\title{
Sidewalk: a RFID Tag Anti-Collision Algorithm Exploiting Sequential Arrangements of Tags
}

\author{
Hyunho Koh, Sangki Yun and Hyogon Kim \\ Korea University
}

\begin{abstract}
Although predicting the RFID tag distribution before a read cycle begins would be generally difficult and even futile, a likely and interesting scenario is where the tags have a sequential arrangement. In large-scale applications such as supply chain management, for instance, hundreds or thousands of the same type of product made by the same manufacturer can be stacked together in the stock. In this paper, we propose a simple RFID tag anti-collision algorithm that exploits such sequential structure of the given tag space to achieve a higher read efficiency. The proposed algorithm is also designed to still achieve the level of performance comparable to that of existing algorithms if tags exhibit little sequential structure. Through exhaustive simulations over various tag distributions, we demonstrate that the proposed algorithm achieves at least $10 \%$ improvement even in the worst case (i.e., when tags are completely randomly distributed), but yields significantly higher improvement when the number of tags and/or the correlation increases.
\end{abstract}

\section{INTRODUCTION}

The RFID tag collision refers to the simultaneous response from multiple RFID tags in response to a read signal from a reader. The consequence is that the reader cannot identify any tag, wasting time, and precious energy in case the reader is battery-operated. The RFID tag collision is an extensively studied problem [1]-[9]. The solution approaches can be classified into two types: tree-based protocols [1]-[7] and aloha-based protocols [8], [9]. The strength of the aloha-based protocols is that it is fast when the number of tags to read is small. However, due to the probabilistic nature it can cause so called the tag starvation problem, and could experience significant performance degradation on large amount of tags [7]. In contrast, the tree-based protocols need to traverse the entire tree space of tag IDs, so can incur relatively long delay. But it is immune to the starvation problem, and has bounded identification delay in face of large number of tags, so it better fits a large-scale RFID system. As the focus of this paper is on large-scale systems, we take the tree-based approach.

In this paper, we propose a simple but efficient tree-based tag anti-collision algorithm, called Sidewalk, that significantly reduces the total read attempts in the initial read cycle. The core idea is to opportunistically exploit the sequential structure of the given tag ID distribution. We believe that in large-scale RFID systems such as supply chain management, hundreds or thousands of the same type of product made by the same manufacturer can be stacked together in the stock. For instance, The EPC code of the product items in such situation will likely have a high correlation among themselves in the "serial number" field. If there is a sequential structure in the RFID tags, the Sidewalk algorithm is designed to exploit it and achieve a significantly higher read performance than existing algorithms. If tag IDs are totally randomly distributed with little sequential structure or there are not many tags, on the other hand, it is designed to still achieve the level of performance comparable to others.

In this paper, we take the Query Tree (QT) algorithm [3] as the baseline for comparison. The reason is that QT algorithm explicitly uses the [prefix of] tag ID itself in the query, which is important to exploit the sequentiality of the tag IDs. Binary tree protocols [], another tree-based approach, does not use the tag ID as the query in the identification process. Thus it is not clear how it can exploit the likely correlative structure of the tag IDs. We defer the investigation of exploiting tag ID correlation under binary tree protocols to future work.

Through exhaustive simulations over various tag distributions, we demonstrate that the proposed algorithm achieves at least $10 \%$ improvement even in the worst case (i.e., when tags are completely randomly distributed), but yields significantly higher improvement when the number of tags and/or the correlation increases.

The rest of the paper is organized as follows. In Section II, we describe the Sidewalk algorithm and the examples of its execution that shed light on different aspects of the algorithm. In Section III, we evaluate the performance of our scheme through extensive simulation. Section IV briefly discuss related work, and Section V concludes the paper.

\section{SIDEWALK}

The core idea of the Sidewalk algorithm starts from the intuition that in many RFID applications the tag arrangement will be serial and sufficiently consecutive. So the first design goal of the Sidewalk algorithm is to exploit this potential correlative structure of the tag whenever possible, without sacrificing the performance when the structure is not found. The second design goal tag anti-collision algorithm that significantly reduces the total number of read attempts in the initial read cycle (a.k.a. frame). Some prior works such as [2] uses the information gathered in the first frame to optimize the reads in subsequent frames if there is need to read the tags repetitively. In our work, however, we focus on optimizing the reads in the first frame. The third design goal of the Sidewalk algorithm is simplicity. The QT algorithm is simple in the sense that it does not demand the tags and the readers to have extra capabilities. In contrast, other protocols may require the tags to be able to detect collision [], or the readers to measure timing of the colliding responses from tags [7]. But the Sidewalk protocol 
does not require of the tags and the readers more than the QT algorithm does.

\section{A. Algorithm description}

The Sidewalk algorithm is described in Algorithm 1. Note that we use a stack as the data structure to store the queries instead of a queue as we perform the depth-first search of the tag ID space. Once the first readable tag is reached, the serial read is attempted across the same level of the tree space laterally (to be precise, to the right). This is why we call the proposed algorithm Sidewalk. In contrast, the breadth-first search of the QT algorithm that uses a queue to store queries would not let us skip the interrogation of the internal nodes of the query tree, which is exactly what the Sidewalk algorithm wants to achieve. In the algorithm description, we refer to the stack used for the depth-first search as the SideWalk Stack (SWS) for convenience.

At the beginning of the algorithm, both SWS and the query to be transmitted, $Q$, are initialized to NULL $(\perp)$. Then the query is attached to 1 and pushed to SWS, i.e., $Q=Q \cdot 1=$ $\perp \cdot 1=1$, followed by the push of 0 . At this point in time, the frame can start. Pop the stack, and we get the first query $Q$, which is transmitted. If a collision occurs, we expand on $Q$ by attaching it to 1 and 0 , and push them to SWS. If the read was successful, however, first the tag ID is stored and then the check is made if the length of $Q,|Q|$, is equal to $|T|$. If so, it means the successful query $Q$ is on the same level with the query on top of SWS ( $T$ ) on the query tree. Namely, the next query is a "side-walk". Note that it cannot be $|Q|<|T|$, since we cannot have gone deeper than $Q$ in the yet unexplored part of the tree. Finally, if $|Q|>|T|, T$ is expanded until it is split into queries on the same read level of $Q$. Note that this step (lines $14-18$ ) is where the lateral traversal, the side-walk, is enforced.

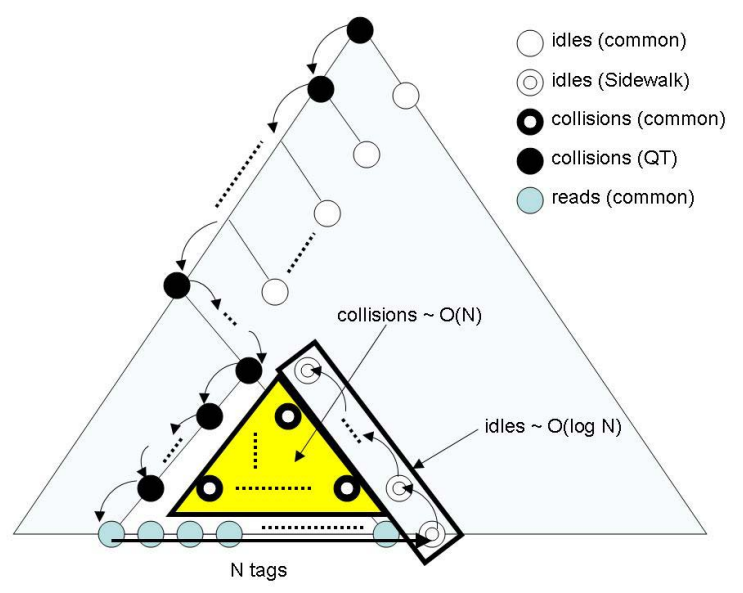

Fig. 1. Collisions vs. idles in Sidewalk.

Why the lateral traversal is important can be conceptually explained through Fig. 1, where the traversal order of the Skywalk algorithm is shown for a completely serial and consecutive tag ID arrangement. Although more complicated collision, read, and idle patterns occur for other distributions, this shows the gist of the idea. The common reads, idles, and

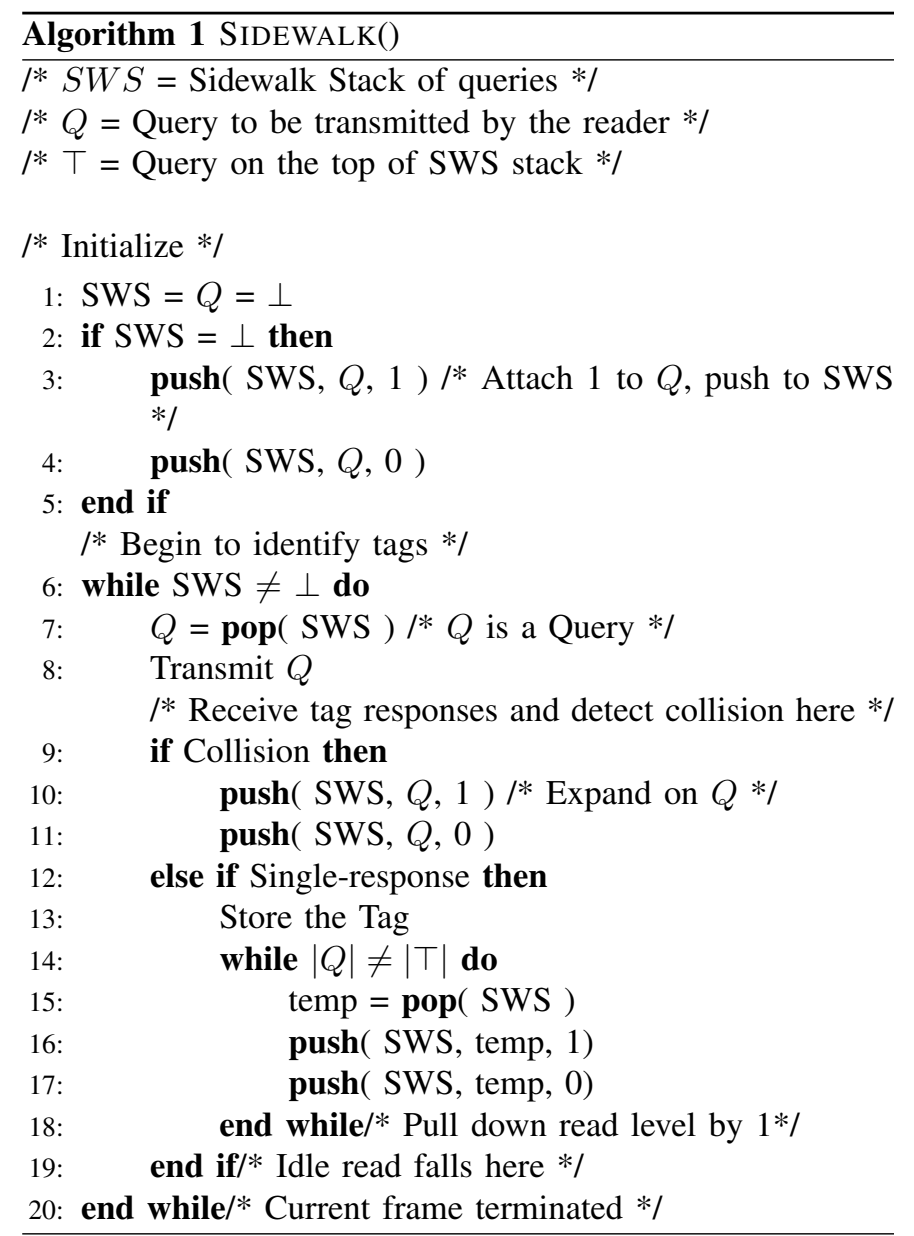

collisions occur for both QT and Skywalk. But executing the Skywalk, some collisions can be avoided (marked by black dots with a hole) while some idles are added (marked by concentric circles) compared to QT. As the gain over QT is given as a function of $N-\log N$, we can see that it will grow with $N$, the number of consecutive tags. Finally, we will see later that the level on which the serial read occurs (which we call the run level) is not necessarily the leaf level.

\section{B. A toy example}

In order to illustrate how the Sidewalk algorithm operates, we take a toy example of tag ID arrangement in Fig. 2. Then,

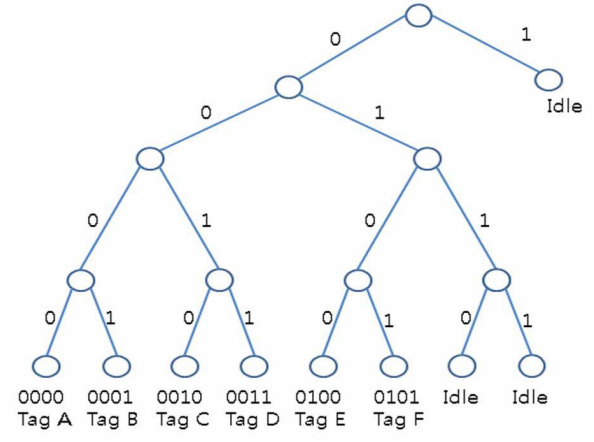

Fig. 2. A toy example of tag ID distribution.

the Sidewalk algorithm executes as follows (also see Table I). 
1) Push 1, 0 to stack (Algorithm 1, lines 1-5).

2) Descend with collisions as in QT. Due to the use of the stack, descending is toward 0000 (lines 9-11).

3) Since the length of the current query and that on the top of the stack is equal, read sequentially (lines 12-13).

4) The lengths differ, so we split the query in the stack until the length agrees (lines 14-18).

5) Read tags C and D without collision.

6) Split query as in Step 4.

7) Read the tags E and F without collision.

8) Again, a query split follows.

9) Finally, the Sidewalk hits an idle. It pops the stack and goes through a series of idles.

10) An idle occurs, which is common to QT. SWS is completely drained. This ends the frame.

TABLE I

EXECUTION OF SIDEWALK ON TOY EXAMPLE

\begin{tabular}{|c|l|l|l|}
\hline Step & Current Query & Response & Sidewalk Stack $\rightarrow \perp$ \\
\hline 1 & & & $\{0,1\}$ \\
\hline 2 & 0 & Collision & $\{00,01,1\}$ \\
\cline { 2 - 4 } & 00 & Collision & $\{000,001,01,1\}$ \\
\cline { 2 - 4 } & 000 & Collision & $\{0000,0001,001,01,1\}$ \\
\hline \multirow{2}{*}{3} & 0000 & Read A & $\{0001,001,01,1\}$ \\
\cline { 2 - 4 } & 0001 & Read B & $\{001,01,1\}$ \\
\hline 4 & & & $\{0010,0011,01,1\}$ \\
\hline 5 & 0010 & Read C & $\{0011,01,1\}$ \\
\cline { 2 - 4 } & 0011 & Read D & $\{01,1\}$ \\
\hline 6 & & & $\{0100,0101,011,1\}$ \\
\hline 7 & 0100 & Read E & $\{0101,011,1\}$ \\
\cline { 2 - 4 } & 0101 & Read F & $\{011,1\}$ \\
\hline 8 & & & $\{0110,0111,1\}$ \\
\hline 9 & 0110 & Idle & $\{0111,1\}$ \\
\cline { 2 - 4 } & 0111 & Idle & $\{1\}$ \\
\hline 10 & 1 & Idle & \{\} \\
\hline
\end{tabular}

For QT, 6 collisions and 2 idles occur, whereas for Sidewalk it is 3 collisions and 3 idles.

\section{Discussion}

The worst-case idle reads overhead of complexity $O(\log N)$ in Fig. 1 occurs when the expansion step (Algorithm 1, lines 14-18) is performed over the subtree boundary. Suppose that the last successful query is $011111 \ldots 1$. The next query is $S W S(\top)=1$. The expansion step expands along the leftmost branch 1 of the query tree, and generates $2|Q|$ queries where $|Q| \leq k$ and $k$ is the depth of the leaf level in the tree, a logarithmic function of the number of tag IDs. Given a large number of tag IDs, this overhead is therefore negligible.

In terms of the run level, the collision pulls it down whereas the idle read pushes it up (Fig. 3(a)). Note that we do not consider a collision to signal the end of a run since the same collision is inevitable in the QT algorithm as well. When the tags are split into many groups, i.e., many runs intervened by idle reads, the idle reads overhead becomes larger and offset the gain made by saving the collisions. Although the gap between the tag IDs may lead to idle reads between them, it depends highly on the specific distribution. For instance, Fig. 3(b) shows that even if the tags appear with many gaps, a run is formed without intervening idle reads. Due to this complication and the unpredictable nature of the real-life tag ID distribution, we turn to simulation for performance evaluation of the Sidewalk in this paper.

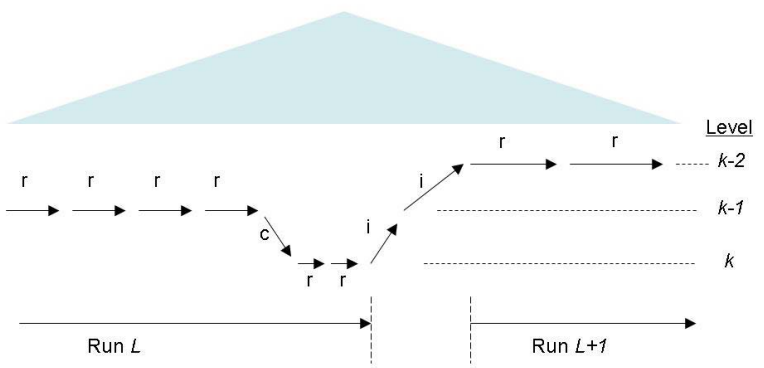

(a) Run level movement according to the type of read result.

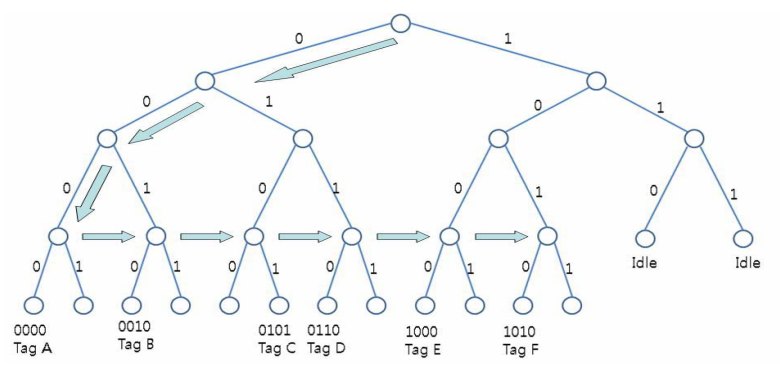

(b) Sidewalk run formed at level $l=k-1$.

Fig. 3. Run level formed above the leaf level $k$.

\section{PERFormance EVAlUATION}

As the specific tag ID arrangement can vary according to the type of application and other factors that we cannot predict yet, we use a generic tag ID generation algorithm that produces tag IDs given two parameters, $p$ and $q$. Specifically, we adopt the Gilbert model since it is frequently used to model bursty binary patterns. In our model, we have two states. The Tag state (denoted by 1 ) is a tag ID generating state. With probability $1-$ $p=P(0 \mid 1)$, we make a transition to the non-tag ID generating state, NoTag (denoted by 0 ). Here, we make a transition to the Tag state with probability $1-q=P(1 \mid 0)$. Starting from some initial tag ID value, we increment the value each time we execute a step in the Gilbert model. Suppose we are in the Tag state at step $k$, and the tag ID that we generated is $I_{k}=$ 001001 . In the next step, we execute the Gilbert model and determine if the tag $I_{k+1}=001010$ will be in our pool of tag IDs to be read. This way, we generate $N$ tags by counting the number of visits to the Tag state to $N$.

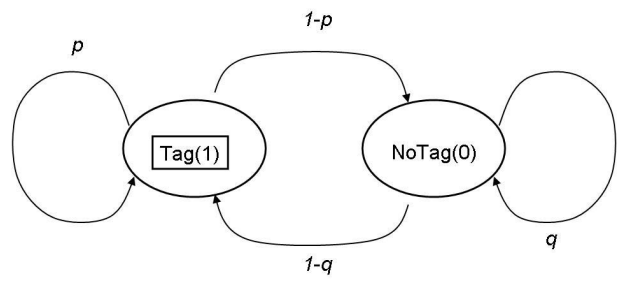

Fig. 4. Tag arrangement generation.

The probability $p$ represents how "sticky" the tags are. Namely, a large $p$ means that once a tag ID appears, it is 
highly likely that a contiguous tag ID is generated next. On the other hand, a high $q$ means the gap between the adjacent tag IDs will be large. In this paper, we experiment with ranges of values of $p$ and $q$ as shown in Table II. Notice that for the symmetric ${ }^{1}$ cases, where $p=q$, the least structure is given for $p=q=0.5$ (not at $p=0.05$ ). If both $p$ and $q$ are low, it is highly likely that the tag IDs alternatingly appear as Fig. 3(b) exemplifies. In the asymmetric cases, $p=0.95, q=0.05$ is the high concentration case and it is likely that the Sidewalk algorithm will most benefit from the structure. In contrast, $p=0.05, q=0.95$ will arrange the tags with small concentrations, large gap intervening between them. The gap size is important because small gaps such as 1 or 2 are sometimes not translated into idle reads (see Fig. 3(b)), elongating the Sidewalk run. As we discussed above, the larger run length translates into higher performance.

TABLE II

TAG ID ARRANGEMENT SETTINGS

\begin{tabular}{|c|c|c|c|}
\hline Experiment & $p$ & $q$ & Characteristics \\
\hline \multirow{4}{*}{ Symmetric } & 95 & 95 & High concentration of tag IDs likely \\
\cline { 2 - 3 } & 80 & 80 & $\uparrow$ \\
\cline { 2 - 3 } & 50 & 50 & No structure \\
\cline { 2 - 3 } & 20 & 20 & $\downarrow$ \\
\cline { 2 - 3 } & 5 & 5 & Alternating tag ID sequence likely \\
\hline \multirow{5}{*}{ Asymmetric } & 95 & 5 & High concentration, small gap \\
\cline { 2 - 3 } & 80 & 20 & $\uparrow$ \\
\cline { 2 - 3 } & 65 & 35 & \\
\cline { 2 - 3 } & 35 & 65 & \\
\cline { 2 - 3 } & 20 & 80 & \\
\cline { 2 - 3 } & 5 & 95 & Low concentration, large gap \\
\hline
\end{tabular}

First, we investigate the symmetric cases. For each configuration, we perform 100 simulation instances to get the averaged data. Although not shown for space, the deviation is negligible, so we only show the average values. Fig. 5 shows the result for $p=q=.5$, in terms of the number of idle cycles, collisions, and total reads of the Sidewalk and QT. In each figure, the horizontal axis is the total number of tags to be read. Notice that this case represents the most random tag ID distribution, the worst configuration for the Sidewalk.

As we expected, the number of idle reads in the Sidewalk is larger than that in the QT. However, the reduction in the collisions is even larger, so that the sum of the idles and collisions is smaller in the Sidewalk. Taking account of the reads common to both schemes, the total cost is still smaller with the Sidewalk. Again, this result is from the least favorable tag ID distributions. Now, we vary $p=q$ as given in Table II and compute the idles+collisions and the total cost. Fig. 6 shows the result.

As predicted, $p=0.5$ yields the least performance gap between the Sidewalk and QT. But as $p$ moves away from 0.5 in either direction, the gap widens. The reason that low $p$ value such as $p=0.05$ leads to a better performance than $p=0.5$ is the structure. In this case, the run level is formed at a lower level (as in Fig. 3(b)), where the Sidewalks may still be achieved across the small gaps. We also notice that as $N$ grows large, the performance gap generally increases. With $N=128$, the total cost reduction is $22 \%(460 \rightarrow 360)$.

\footnotetext{
${ }^{1}$ The symmetric-asymmetric distinction is for convenience.
}

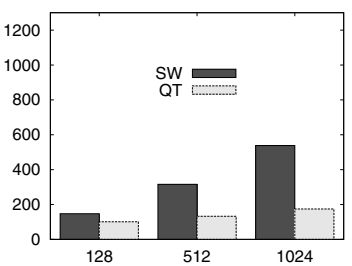

(a) Idles.

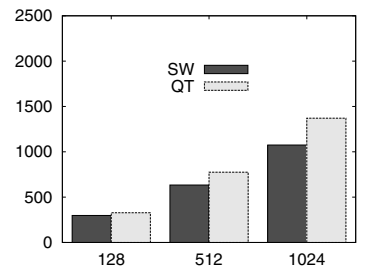

(c) Idles + collisions.

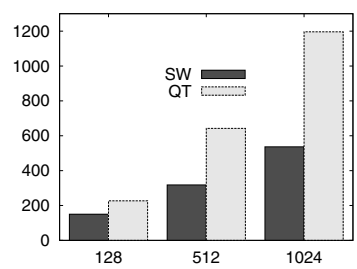

(b) Collisions.

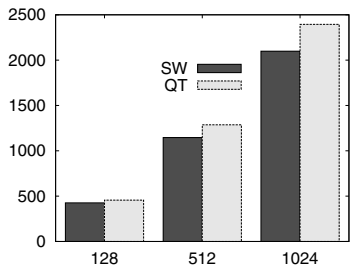

(d) Idles + collisions + reads.
Fig. 5. Performance comparison between Sidewalk and QT under symmetric configuration, $p=q=0.5$.

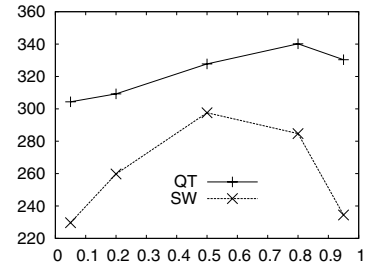

(a) Idles+Collisions, $N=128$.

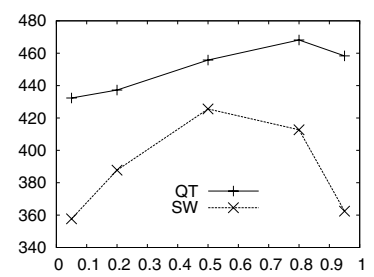

(c) Total, $N=128$.

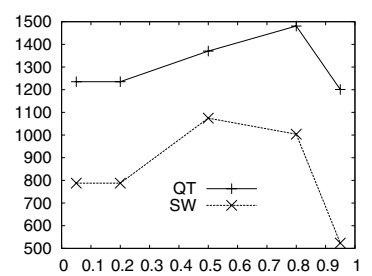

(b) Idles+Collisions, $N=1024$.

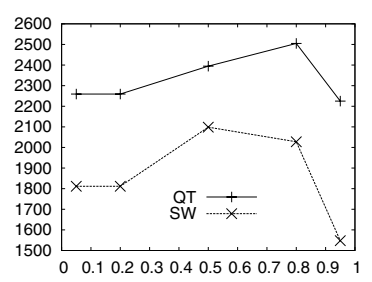

(d) Total, $N=1024$.
Fig. 6. Performance comparison between Sidewalk and QT under symmetric configuration, $p=q$.

With $N=1024$, on the other hand, the reduction is $32 \%$ $(2210 \rightarrow 1530)$

Fig. 7 is a typical performance result for asymmetric cases. Here, we set $p=.2, q=.8$. Again, the Sidewalk has larger number of idles, whereas QT causes even larger number of collisions. Looking at the broader spectrum of $p=1-q$, we notice that both the Sidewalk and the QT improve as $p$ increases in Fig. 8. When $p$ is small, the Sidewalk performance is comparable to, or even slightly lower than, that of QT. But this is limited to the cases with small $p$. Recollect that our design goal was to achieve comparable performance in the worst case distributions of the RFID tags, but exploit whatever sequential structure there is to attain higher performance. As we intended, Fig. 8 shows that the performance gap widens as $p$ grows. Here, the QT algorithm produces increasingly marginal performance improvement whereas the Sidewalk algorithm cost almost linearly decreases as $p$ increases.

\section{RELATED WORK}

One of the representative tree-based approaches is querytree $(\mathrm{QT})$ protocol that uses a prefix of ID as a query. In the 


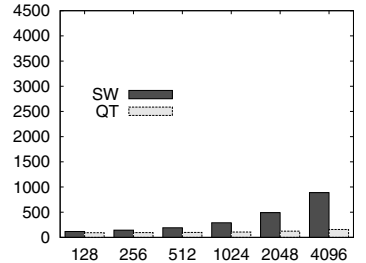

(a) Idles.

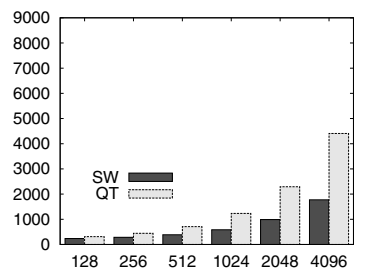

(c) Idles + collisions.

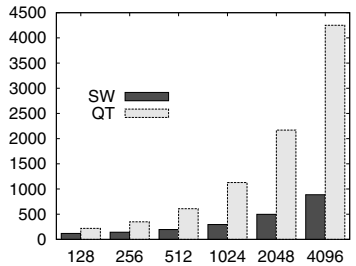

(b) Collisions.

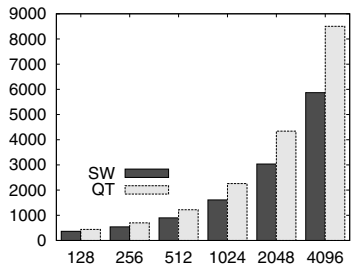

(d) Idles + collisions + reads.
Fig. 7. Performance comparison between Sidewalk and QT under asymmetric configuration, $p=0.8, q=0.2$.

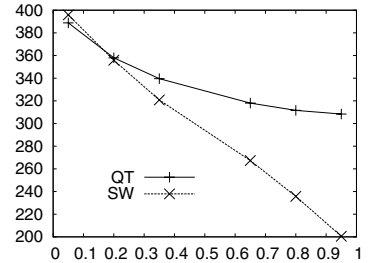

(a) Idles+Collisions, $N=128$.

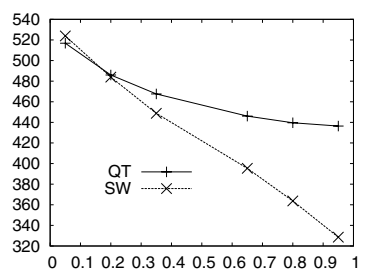

(c) Total, $N=128$.
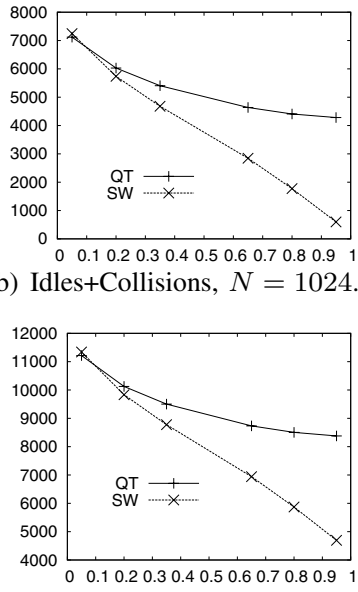

(d) Total, $N=1024$. (b) Idles+Collisions, $N=1024$

Fig. 8. Performance comparison between Sidewalk and QT under symmetric configuration, $p=q$.

query-tree protocol, the reader maintains a query queue which contains prefixes of ID. When a prefix is transmitted, the tags that have same ID prefix with the query respond to it. If there is tag collision, the reader extends the prefix by adding 0 and 1 and retries. The tag identification process finishes when the reader queue becomes empty.

Although the query-tree protocol finishes its identification process in a bounded time, it can experience many collisions when the number of tags is large. Adaptive Query Splitting protocol (AQS) [2] memorizes the read results (collisions, reads, no responses) in the first read cycle in a separate queue, and utilizes it in subsequent read cycles if repetitive reads of the tag space are required.

Choi et al. proposes a modification of the QT protocol that exploits the characteristic of EPC Class 1 Gen. 2 in order to improve its performance in the initial read cycle [4]. Each tag generates a 16-bit random number as a temporary ID as it is more efficient than working with a 96-bit tag. Then the QT algorithm is applied to the short ID. Chiang et al. [5] proposes an essentially similar idea. In this scheme, the tags also generate a random, short prefix. But the difference is that if the randomly generated prefixes collide, it is the tags that should detect the collision and lengthen the prefix by attaching 0 or 1 to it.

Bhandari et al. considers an environment where the products may come from same manufacturer and may have same product IDs, such as exclusive showrooms, shipment points of big malls. The proposed scheme, IQT, exploits the tags that share a common prefix so that it can eliminate collisions correspondingto the traversal of the common prefix in the query tree [6]. Once the common prefix is figured out, the QT algorithm is applied to the suffix portion of the IDs.

Ryu et al. improves on both the initial read cycle and AQS [7]. Unlike the QT algorithm, when collision occurs this scheme splits the prefix using the 4-ary tree. While reducing collisions, this method can increase the idle reads due to increased branching. In order to reduce the possibility of idle reads, the reader analyzes the temporal pattern of the responses in time, and estimates the number of existing tags.

\section{CONCLUSION}

In this paper, we propose a novel tag assignment scheme that minimizes the energy consumption of the reader in a large scale RFID system. Our scheme focuses on reducing the number of idle cycles of a reader when assigning a tag, and the assignment algorithm is efficient and does not incur much overhead. Performance evaluation shows that our scheme can significantly reduce the number of idle cycles in a frame, and it can decrease the energy consumption by more than $20 \%$ compared with the random tag assignment approach.

\section{ACKNOWLEDGEMENT}

This work was supported by grant No. R01-2006-00010510-0 from the Basic Research Program of the Korea Science \& Engineering Foundation.

\section{REFERENCES}

[1] EPCglobal. Class 1 Generation 2 UHF Air Interface Protocol Standard Version 1.0.9, 2005.

[2] J. Myung and W. Lee, "Adaptive Splitting Protocols for RFID Tag Collision Arbitration," in Proc. ACM MOBIHOC, pp. 202-213, Florence, Italy, May, 2006

[3] C. Law, K. Lee and K.-Y. Siu, "Efficient memoryless protocol for tag identification," Proc. 4th Int'l Workshop Discrete Algorithms and Methods for Mobile Computing and Communications, pp. 75-84, Boston, Massachusetts, USA, Aug. 2000.

[4] Ji Hwan Choi; Dongwook Lee; Hyuckjae Lee; Query tree-based reservation for efficient RFID tag anti-collision Communications Letters, IEEE Volume 11, Issue 1, Jan. 2007 Page(s):85 - 87

[5] Kong Wa Chiang, Cunqing Hua, Tak-Shing Peter Yum, PrefixRandomized Query-Tree Protocol for RFID Systems, Communications, 2006. ICC '06. IEEE International Conference on Publication Date: June 2006, Volume: 4, On page(s): 1653-1657

[6] Bhandari, Naval; Sahoo, Anirudha; Iyer, Sridhar, Intelligent Query Tree (IQT) Protocol to Improve RFID Tag Read Efficiency Information Technology, 2006. ICIT '06. 9th International Conference on 18-21 Dec. 2006 Page(s):46 - 51.

[7] Jiho Ryu, Hojin Lee, Yongho Seok, Taekyoung Kwon and Yanghee Choi, A Hybrid Query Tree Protocol for Tag Collision Arbitration in RFID systems, communications, 2007. ICC '07. IEEE International Conference on 24-28 June 2007 Page(s):5981 - 5986

[8] H. Vogt, "Efficient object identification with passive RFID tags," Proc. Int'l Conf. Pervasive computing, LNCS 2414, pp. 98-113, Apr. 2002.

[9] J. Zhai and G. Wang, "An anti-collision algorithm using twofunctioned estimation for RFID tags," Proc. Int'l Conf. Computational Science and its Applications, May 2005. 